\title{
4th International Conference on Bronchoalveolar Lavage, organized by the European BAL Task Group
}

\author{
Umeå, June 17-19, 1993 \\ H. Klech* \\ Chairman European BAL Task Group
}

Bronchoalveolar lavage (BAL) is today a widely accepted tool both for pulmonary research and for selected clinical purposes [1-3]. BAL is now used in experimental medicine, in occupational medicine, by infectious disease specialists, by intensive care specialists, and by pulmonary physicians.

The 4th International Conference on Bronchoalveolar Lavage was an excellent forum to review topics for which BAL is used or will be used in the future. The local organizers were L. Bjermer and T. Sandström from Umeå University.

\section{BAL in children}

\section{(organized by G. Rossi and R. Ronchetti)}

For the first time, a comprehensive review was given concerning the use of BAL in children. European experiences were presented by Ronchetti and Midulla, complemented by American experiences presented by Wood. Midulla revealed technical aspects of BAL. His data demonstrated that in children fractional processing of BAL can differentiate between bronchial and alveolar lavage, and that these two samples differ in the concentration of lymphocytes, neutrophils, total protein, albumin, fibronectin and hyaluronic acid. He also presented data on reference values in "normal" children, showing that the differential cell count of BAL in the paediatric age group is quite similar to the count in normal adults.

Ronchetti presented data concerning cellular and noncellular BAL components in children with interstitial lung disease (ILD). These children had a high concentration of lymphocytes, fibronectin and hyaluronic acid. These data demonstrated that in children with ILD, BAL is a good tool for use both in diagnosis and follow-up.

Clements presented data obtained from children with sarcoidosis, and demonstrated that in children BAL can also be used as a research tool. The application of BAL in the diagnosis of opportunistic infections in immunocompromised children was presented by de Blic. Finally, Dab and Grigg presented their experiences with BAL in neonates. Their presentations supported the concept that BAL is also a safe and well-standardized technique for use in neonates.

Correspondence: ${ }^{*} \mathrm{H}$. Klech, Ludwig Boltzmann Institute for BAL, 2nd Med Dept Wilhelminenspital, A-1171 Vienna, Austria.
BAL in oncology

(organized by St. Rennard and R. Hendriksson)

In their lectures, Rennard, Hendrikson and Pirozynski concluded that BAL can be helpful in diagnosis of lung cancer, and is adequately complementary to other diagnostic techniques. The important factor is that just a couple of malignant cells obtained by BAL can provide the diagnosis. There was a comprehensive review concerning the role of BAL for assessment of immunological and cellular changes due to cancer in the pulmonary environment by Semenzato, emphasizing that BAL can add to the further management of patients with lung cancer.

Obstructive lung disorders

(organized by M. Saetta and M. Söderberg)

A significant part of the conference was dedicated to the question of how BAL can contribute to the understanding and management of obstructive lung disorders. Although it was concluded that BAL is not helpful for the daily management of these conditions, it was clear that BAL can be applied in interesting research topics concerning the pathophysiology of chronic obstructive pulmonary disorders; thus, helping to gain further insight into the underlying pathogenic mechanisms and the role of cells and enzymes.

\section{Interstitial lung disorders (organized by P. Haslam and A. Eklund)}

Haslam introduced the session and emphasized that interstitial lung disorders (ILDs) were historically the first group of lung diseases to be investigated exhaustively by the use of BAL. Moreover, they still account for a third of all current publications on human BAL studies. This wealth of information has firmly established the importance of BAL as an aid to diagnosis and for research investigations of pathogenic mechanisms. However, there has been no major breakthrough which has substantially improved the management or treatment of these serious chronic lung diseases. We still do not fully understand why the rates of disease progression are 
so variable in different ILDs, and in different individuals with the same disease. The need to obtain a better understanding of the factors associated with disease progression and resolution remains one of the most important key areas for current research. This was chosen as the focus for the remainder of the session.

Elias presented a superb state of the art review of cytokines and their synergistic interactions in basic mechanisms of lung injury and repair. He emphasized that cytokine mediators can be produced by many tissue cell types. Informed discussion of cytokines cannot, therefore, be restricted to information gained solely by BAL. He mentioned the early work from his group, which first demonstrated that fibroblast proliferation can be enhanced by interleukin-1 (IL-1) or tumour necrosis factor- $\alpha$ (TNF$\alpha$ ), but, by contrast, when acting together these cytokines synergistically inhibit fibroblast proliferation. He then presented subsequent work, showing that fibroblasts themselves can produce cytokines, including the proinflammatory mediator interleukin-6 (IL-6); and that IL-6 production can be regulated by IL- 1 and TNF- $\alpha$, which together stimulate production but when acting alone are inhibitory. His group has also, very recently, shown that IL-1 can act synergistically with another cytokine interleukin-11). Of possible relevance to the pathogenesis of sarcoidosis, the group has recently also demonstrated IL-11 in the centre of giant cells and messenger ribonucleic acid (mRNA) for IL-11 in the skin of patients with sarcoidosis, and has shown that incubation of monocytes with IL-11 induces giant cell formation. IL-11 and IL-6 are both T-lymphocyte-dependent cytokines, which have stimulatory effects on B-lymphocytes.

Elias then turned to consideration of the role of cytokines in the resolution of tissue inflammation. He mentioned that tumour growth factor- $\beta$ (TGF $\beta$ ) for example, whilst stimulating fibrogenesis can inhibit many immune responses; and interferon- $\gamma$ (IFN- $\gamma$ ), whilst a macrophage activator and capable of stimulating the growth of resting fibroblasts, can inhibit the proliferation of growing fibroblasts and can also inhibit the activation of the Th2 subset of lymphocytes, which control the development of humoral immune responses; whilst yet another cytokine, interleukin-10 (IL-10), can inhibit the activation of the Th1 subset of lymphocytes, which control the development of cell-mediated immunity. Finally, he drew attention to another important issue, namely cytokine regulation of lung epithelial cells. He also reported the very recent finding that lung fibroblasts can produce hepatocyte growth factor, which can stimulate epithelial cell regeneration; and that production can be stimulated by IL- 1 and TNF- $\alpha$ but totally inhibited by TGF- $\beta$. Moreover, increased levels of hepatocyte growth factor have been detected in BAL samples in idiopathic pulmonary fibrosis (IPF) and sarcoidosis. Finally, Elias reported the new observation that lung epithelial cells contain a novel tyrosine kinase receptor that regulates their proliferation.

Prior spoke about evidence that he has obtained in patients with chronic pulmonary sarcoidosis, which indicates that patients with higher pretreatment levels of BAL lymphocytes and higher circulating levels of the lymphokine IFN- $\gamma$ have a more favourable long-term prognosis and response to steroid therapy; being more likely to achieve complete radiographic clearing and less likely to relapse than patients with lower levels of IFN$\gamma$. He also reported that a small number of patients with the more fibrotic cryptogenic fibrosing alveolitis also have minor elevations in serum IFN- $\gamma$, and that these patients were also more likely to be steroid responsive. Basic research studies by Elias and others have shown that IFN- $\gamma$ can markedly inhibit the growth of fibroblasts in vitro. In view of these observations, Prior suggested that IFN- $\gamma$ may contribute to the regulation of fibrogenesis in vivo and be of potential value as a therapeutic agent in fibrosing lung diseases. In support of this suggestion, he drew attention to encouraging results which have been obtained in a recent preliminary trial of recombinant IFN- $\gamma$ in patients with fibrosing alveolitis in association with scleroderma.

Eklund gave a general overview focusing upon the interpretation of BAL in relation to tobacco smoke exposure. In particular, he drew attention to the smokingrelated phenomenon of autofluorescence in macrophages.

Bjermer then spoke about the clinical effects of smoking, noting that in some instances smoking appears to increase susceptibility for diseases, for example in IPF (syn. cryptogenic fibrosing alveolitis) and histiocytosis $\mathrm{X}$; whereas, in other diseases, there is evidence that smoking reduces susceptibility, for example in extrinsic allergic alveolitis (syn. hypersensitivity pneumonitis) and pulmonary sarcoidosis. Among breast cancer patients receiving radiation treatment, smokers also appear less susceptible to the development of radiation pneumonitis, although more prone to metastases. He then expanded this topic by describing the results of experiments in a rat model of radiation pneumonitis showing that exposure to cigarette smoke (20-25 cigarettes $\cdot$ day $\left.^{-1}\right)$ markedly reduced the development of inflammatory responses, more notably in the pulmonary interstitium than in the perivascular and peribronchial areas.

Nagai then concluded the session by presenting recent data expanding basic information on the in vivo effects of smoking on BAL cells in healthy controls. She reported that an age-related increase in the ratio of CD4/CD8 T-lymphocyte subsets is seen both in BAL and blood from nonsmokers and ex-smokers, but is not seen in current cigarette smokers. This was due to a decrease in CD4 cells in smokers. Messenger RNA for IL-1 receptor antagonists, a homeostatic regulator of IL-1, was found to be decreased in BAL cells from healthy smokers, and from smokers with IPF and sarcoidosis, compared with healthy nonsmokers. This suggests that a smokingrelated deficiency of IL-1 receptor antagonist and failure to adequately regulate IL-1 may be one of the many effects involved in smoking-related modulation of disease.

Environmental lung disorders (organized by U. Costabel and T. Sandström)

Sandström reviewed the BAL studies that had been made so far in humans before and after exposure to nitrogen dioxide $\left(\mathrm{NO}_{2}\right)$, sulphur dioxide $\left(\mathrm{SO}_{2}\right)$ and diesel 
exhaust. It was reported that these different exposures led to different kinds of inflammation depending on the deposition and chemical effects of the various components. It was evident that smokers, in comparison with nonsmokers, reacted with an enhancement of their already existing alveolar macrophage inflammation, but did not develop any acute lymphocytic inflammation. Exposure to diesel exhaust caused far more alveolar macrophage effects, including suppression of the phagocytic capability, in comparison with $\mathrm{NO}_{2}$ alone. This was suggested to be due to particulates and carbohydrates.

Koren demonstrated that ozone has far more pronounced inflammatory effects compared to $\mathrm{NO}_{2}$, another oxidant gas. Ozone caused a pronounced polymorphonuclear neutrophil (PMN) increase in BAL fluid of humans, together with an increase in IL-6, interleukin-8 (IL-8), fibronectin and protein concentrations. The effect of repeated exposure to ozone was presented in a very complex study with BAL after air, one hour after a 5 day period of ozone exposure, and 10 and 20 days after ozone exposure. Attenuation of most parameters was seen after repeated exposure, compared with previous single exposure studies. Signs of tissue damage in terms of, e.g. increase in lactate dehydrogenase, (LDH) were, however, not attenuated.

von Essen presented a review of the effects of organic dust exposure. The BAL findings during acute dairy farmers' lung disease, with increase of lymphocytes and mast cells, and a decrease of the CD4/CD8 ratio, together with an increase in hyaluronan and procollagen-3peptide were discussed. It was shown that, during harvest, grain farmers had an increase in neutrophils and lymphocytes, together with elevations in mRNA levels for IL-8 and monocyte chemoattractant protein-1 (MCP1). In the separate entity, organic dust toxic syndrome, BAL fluid was shown to be dominated by an increase in neutrophils.

Costabel reviewed the immunological aspects of mineral dust induced ILDs, such as asbestosis and silicosis. Animal studies are sometimes in conflict with data obtained from humans. In vivo and in vitro effects may also show different results. In addition, the smoking status may have a significant effect. To summarize the data, after inhalation of particles and fibres, alveolar macrophages become activated and release proinflammatory mediators, such as IL- 1 , TNF- $\alpha$, and IFN $\gamma$. By secretion of chemoattractants, they also induce an influx of neutrophils and lymphocytes to the lung; the CD4/CD8 ratio being low after silica exposure and high after asbestos exposure, especially in those individuals with milder disease on chest radiography. Whether it is true that the increase in BAL lymphocytes plays a protective role against the progression of disease, or the opposite, is under debate. The mechanisms involved in the process are not well understood, nor the potentially predictive factors which would recognize those patients prone to develop severe fibrosis.
Teschler reported on the assessment of asbestos bodies (ABs) and fibres in BAL. Several studies have indicated that the upper limit of normal for $\mathrm{ABs}$ is approximately $0.5-1.0$ per $\mathrm{ml}$ BAL fluid. There is a rough correlation between the concentration of $\mathrm{ABs}$ in $\mathrm{BAL}$ and in the lung tissue: one $\mathrm{AB} \cdot \mathrm{ml}^{-1} \mathrm{BAL}$ corresponds to about 1,000 $\mathrm{AB} \cdot \mathrm{g}^{-1}$ wet lung tissue. The content of uncoated asbestos fibres in BAL, as assessed by electron microscopy, and its clinical significance is under current investigation. The normal threshold appears to be 1,000 uncoated fibres $\cdot \mathrm{ml}^{-1} \mathrm{BAL}$. It was stressed that elevated $\mathrm{AB}$ counts in BAL are merely an indication of exposure and not of disease, which has to be proven by other means. On the other hand, a BAL specimen negative for $\mathrm{AB}$ cannot exclude enhanced occupational exposure to asbestos.

\section{Technical aspects}

\section{(organized by H. Klech and L. Bjermer)}

There was an interesting review concerning age and racial differences in BAL results, followed by an excellent review by Walters about the value of soluble components in BAL. It reflected the difficulty in obtaining accurate measurements of the epithelial lining fluid by use of soluble denominators or markers, such as albumin or urea. Although these techniques are widely applied in scientific and research considerations, those elements are so far not reliable in order to make decisions on clinical grounds. The application of molecular biological tools on BAL fluid and BAL cells is the most promising technique, which will provide us with more insights into common and new disorders of the lung. The easy access to pulmonary cells and fluids by means of bronchoalveolar lavage makes the application of these techniques extremely rewarding.

\section{Outlook}

The 5th International Conference will take place in Dublin in 1995; it will be jointly organized by L. Poulter (London) and C. O'Burke (Dublin).

\section{Reference}

1. Klech H, Pohl W, eds. Clinical guidelines and indications for bronchoalveolar lavage (BAL) Report of the European Society of Pneumology Task Group on BAL.. Eur Respir J 1990; 3: 937-974.

2. Klech H, Hutter C, eds. Technical recommendations and guidelines for bronchoalveolar lavage (BAL). Report of the European Society of Pneumology Task Group on BAL. Eur Respir J 1989; 2: 561-585.

3. American Thoracic Society. Clinical role of bronchoalveolar lavage in adults with pulmonary disease. Am Rev Respir Dis 1990; 142: 481-486. 